\title{
Diagnostic performance of the noninvasive prenatal FetoGnost RhD assay for the prediction of the fetal RhD blood group status
}

\author{
Tobias J. Legler ${ }^{1} \mathbb{D} \cdot$ Sandra Lührig ${ }^{1} \cdot$ Irina Korschineck ${ }^{2} \cdot$ Dieter Schwartz $^{3}$
}

Received: 19 August 2020 / Accepted: 27 March 2021 / Published online: 9 April 2021

(c) The Author(s) 2021

\begin{abstract}
Purpose To evaluate the diagnostic accuracy of a commercially available test kit for noninvasive prenatal determination of the fetal RhD status (NIPT-RhD) with a focus on early gestation and multiple pregnancies.

Methods The FetoGnost RhD assay (Ingenetix, Vienna, Austria) is routinely applied for clinical decision making either in woman with anti-D alloimmunization or to target the application of routine antenatal anti-D prophylaxis (RAADP) to women with a RhD positive fetus. Based on existing data in the laboratory information system the newborn's serological $\mathrm{RhD}$ status was compared with NIPT RhD results.

Results Since 2009 NIPT RhD was performed in 2968 pregnant women between weeks $5+6$ and $40+0$ of gestation (median $12+6)$ and conclusive results were obtained in $2888(97.30 \%)$ cases. Diagnostic accuracy was calculated from those 2244 (77.70\%) cases with the newborn's serological RhD status reported. The sensitivity of the FetoGnost RhD assay was $99.93 \%$ (95\% CI 99.61-99.99\%) and the specificity was $99.61 \%$ (95\% CI 98.86-99.87\%). No false-positive or false-negative NIPT RhD result was observed in 203 multiple pregnancies.

Conclusion NIPT RhD results are reliable when obtained with FetoGnost RhD assay. Targeted routine anti-D-prophylaxis can start as early as $11+0$ weeks of gestation in singleton and multiple pregnancies.
\end{abstract}

Keywords NIPT-RhD $\cdot$ Diagnostic accuracy $\cdot$ Targeted anti-D prophylaxis $\cdot$ Multiple pregnancies

\section{Introduction}

In alloimmunised pregnant women non-invasive prenatal testing (NIPT) can be applied to identify fetuses with increased risk due to the presence of at least one blood group specific nucleotide polymorphism (SNP) [1]. Since large-scale NIPT for RhD studies have been performed, the published confidence intervals for diagnostic sensitivity and specificity are relatively small. In contrast, smaller patient cohorts have been investigated to validate NIPT for other blood groups namely Kell, $\mathrm{RhC}$, Rhc and $\mathrm{RhE}$ resulting in a very similar diagnostic accuracy compared with NIPT for

Tobias J. Legler

tlegler@med.uni-goettingen.de

1 Department of Transfusion Medicine, University Medical Center Göttingen, Robert-Koch-Str. 40, 37075 Göttingen, Germany

2 Ingenetix GmbH, Vienna, Austria

3 Department of Blood Group Serology and Transfusion Medicine, Medical University of Vienna, Vienna, Austria
$\mathrm{RhD}$ but with higher confidence interval [2-4]. To date, real-time PCR is the most common technology applied for the determination of fetal blood groups [1]. More recently, droplet digital PCR and next-generation sequencing have been proposed for noninvasive fetal molecular blood group genotyping, especially when antigens different from $\mathrm{RhD}$ have to be investigated [5-7]. Theoretically, compared with real-time PCR, the accuracy could be higher with these more modern methods. However, larger cohort studies still have to be done to provide evidence for this consideration.

It is very well established for over 50 years that the risk of Rhesus $\mathrm{D}(\mathrm{RhD})$ alloimmunisation and the number of subsequent cases of hemolytic disease of the fetus and newborn (HDFN) can be reduced by postpartal and routine antenatal anti-D prophylaxis (RAADP) [8-10]. However, a considerable number of anti-D-immunoglobulin doses are applied unnecessarily in pregnancies with $\mathrm{RhD}$ negative fetuses. Because of the scarce supply of anti-D immunoglobulin and possible adverse reactions there has always been the goal to restrict this treatment to women carrying $\mathrm{RhD}$ positive fetuses only (targeted RAADP). 
Since the feasibility of targeted antenatal RAADP based on the result from a NIPT for the prediction of the fetal $\mathrm{RhD}$ status has been proposed by Dennis Lo's group and a Dutch group in 1998 it took about 12 years until the first nationwide program for targeted RAADP was implemented in Denmark [11-13]. Until today many validation studies have been published and extensively reviewed which revealed an excellent diagnostic accuracy of NIPT for RhD [14-21]. It has to be stressed in the given context that only false-negative NIPT $\mathrm{RhD}$ results may have relevant consequences (i.e., increased risk for anti-D alloimmunisation). False-positive results will just lead to unnecessary anti-D immunoglobulin administration in such cases as without any NIPT RhD testing.

Despite extensive literature dealing with the diagnostic accuracy of NIPT assays already used in diagnostic laboratories for the prediction of the fetal RhD status, we identified the following three research questions which we address in this paper:

1. What is the diagnostic accuracy of a new commercially available real-time PCR assay?

2. Can targeted RAADP be applied as early as week $11+0$ of gestation (wg)?

3. Is targeted RAADP applicable to multiple pregnancies?

\section{Methods}

The FetoGnost RhD real-time PCR assay (Ingenetix GmbH, Vienna, Austria), not CE IVD approved yet, was evaluated by reviewing NIPT-RhD results and $\mathrm{RhD}$ blood group serology results from newborns from the Medical University of Vienna in a retrospective analysis. The study protocol was approved by the local ethical committee (approval no. 1927/2020). Oligonucleotides and probes for $R H D$ exon 5 (VIC) and 7 (FAM) verified in a multicenter study were used with minor modifications [22, 23]. Exon 5 and 7 reagents were used in one multiplex PCR together with primers/probes for $R H D$ exon 10 (NED) and a 76 bp synthetic oligonucleotide as internal positive control (IPC, Cy5). For real-time PCR instruments which lack the filter for $\mathrm{Cy} 5$, the IPC can also be tested with a VIC-labelled probe in a separate tube. The IPC target was added to the extraction tube to detect DNA extraction as well as amplification failures. IPC primer-binding sites were specific for mus musculus ICAMI, whereas the IPC probe binding site was specific for human $G A P D H$. Every sample was tested in triplicates resulting in a maximum of $9 R H D$ specific calls. Samples with 0-2 calls were interpreted as $\mathrm{RhD}$ negative, 7-9 calls as $\mathrm{RhD}$ positive, 3-6 calls as inconclusive.

In Vienna routine, NIPT-RhD has been performed since 2009 after in-house validation using 50 samples provided by the Göttingen group pursuant to guideline $98 / 79 / \mathrm{EG}$ of the European Commission for clinical decision making in all $\mathrm{RhD}$ negative pregnant women, either to advice the management in cases with anti-D alloimmunization or to apply targeted RAADP.

In general, all pregnancies at the Vienna Medical University's Obstetrics department are considered to have some sort of risk, at least at some time during the course of pregnancy. Blood group data from all live births at this department have continuously been checked with NIPT RhD test results as NIPT RhD testing performance evaluation. Since a significant proportion of pregnancies were released from risk management and delivered in another hospital or pregnancies resulted in no live birth, there has been a relatively high number of NIPT RhD cases without a corresponding newborn's RhD typing result in our lab (lost to follow-up).

All samples were collected locally, after informed consent was obtained, using $8 \mathrm{ml}$ K2EDTA separator tubes (Ref 455021, Greiner Bio-One GmbH, Austria). Plasma was separated by centrifugation $10 \mathrm{~min}(4000 \mathrm{RCF})$ and the original tubes were stored at $-20{ }^{\circ} \mathrm{C}$ within $6 \mathrm{~h}$ after venipuncture until analysis. Cell-free fetal DNA was extracted manually using a spin column method (QIAamp DSP Virus Kit, Qiagen, Hilden, Germany) in the first years and since 2017 automatically (Maxwell, RSC ccfDNA Plasma Kit, Promega GmbH, Walldorf, Germany). Real-time PCR (StepOne Plus, Applied Biosystems, Foster City, CA, US) was performed according to the FetoGnost RhD handbook. In addition, an $S R Y$ sequence was detected as the fetal marker. In brief, primers RhD SRY-1F TGGCGATTAAGTCAA ATTCGC (10 pmol/30 $\mu \mathrm{l} \mathrm{PCR),} \mathrm{RhD} \mathrm{SRY-72R} \mathrm{CCCCCT}$ AGTACCCTGACAATGTATT (10 pmol/30 $\mu \mathrm{l} \mathrm{PCR})$ and TaqMan ${ }^{\circledR}$ probe RhD SRY-30 T NED-CCTGACTGCTCT ACTGC-MGB (RevComp, $5 \mathrm{pmol} / 30 \mu \mathrm{l}$ PCR) were used to amplify part of the Y-chromosome in male fetuses. Furthermore, hypermethylated RASSF $1 A$ was analyzed as a second, sex-independent fetal marker according to the protocol previously described by Chan et al. [24]. One thermocycler profile was used for all real-time PCR assays. Following an incubation at $50{ }^{\circ} \mathrm{C}$ for 2 min and $95{ }^{\circ} \mathrm{C}$ for 20 s 60 2-step cycles with denaturation at $95^{\circ} \mathrm{C}$ for $5 \mathrm{~s}$ and $60{ }^{\circ} \mathrm{C}$ for $1 \mathrm{~min}$ were performed.

Negative (aq. dest.) and positive (plasmid containing target sequences for RHD, SRY, RASSFIA and ACTB) controls have been included in each run. In addition, for each new lot of the assay a backup sample from a previous NIPT RhD testing with a confirmed follow-up of a $\mathrm{RhD}$ positive male child has been tested.

In general, the Vienna Immunohaematology laboratory has recommended the confirmation of negative NIPT RhD on a second sample (independent venipuncture) before any consequences (omission of anti-D immunoglobulin in non-immunized women, cessation of risk management in immunized women) are drawn. Because there was no single 
false-negative testing observed in the first 6 years of routine testing and of economic constraints, this recommendation was not stringently followed thereafter. One false-negative typing in a non-immunized woman that occurred in 2016 would most probably have been prevented if this strategy had been maintained. For this study, only the first testing results were included in the analysis.

Statistical analysis concerning calculation of confidence interval (CI, Wilson interval) using R (version 4.0.2 (202006-22) was performed in collaboration with the Department of Medical Statistics (University Medical Center Göttingen).

\section{Results}

The diagnositic accuracy of the FetoGnost RhD assay was determined from the continuous performance evaluation of routine testing. NIPT-RhD together with controls for hypermethylated $R A S S F 1 A$ as well as $S R Y$ was performed in 2968 pregnancies at week $5+6$ until week $40+0$ of gestation (median $12+6 \mathrm{wg}$ ). About half of the samples (50.9\%) were collected in the first trimester. Patients were between 16 and 50 years (median 32 years). Conclusive results were obtained in 2888 (97.30\%) cases (Fig. 1). Because rather low ct-values were observed and a maternal $R H D$ positive genotype was suspected ( $n=15$ ), or the number of $R H D$ specific calls were not conclusive $(n=29)$ or $1-2$ exons did not show any positive result and a D-variant in the fetus was suspected $(n=9)$, or the $R H D$ result was negative, but the fetal controls were negative or inconclusive $(n=27)$, no conclusive NIPT RhD results was reported in 80 (2.70\%) cases. Among these 27 NIPT RhD negative cases with inconclusive fetal markers, there was one at $11+3 \mathrm{wg}$ with 7 of 9 negative reactions in the FetoGnost RhD test, where the fetus was tested $\mathrm{RhD}$ positive after birth, 21 newborns were $\mathrm{RhD}$ negative and in 5 cases no result from a newborn was available.

In 2244 of 2888 (77.70\%) cases with conclusive NIPT $\mathrm{RhD}$ result, information about the RhD phenotype of the newborns was available from the immunohematology laboratory. If at least one child was $\mathrm{RhD}$ positive or weak $\mathrm{D}$ in multiple pregnancies, this was counted as "one $\mathrm{RhD}$ positive newborn" in Fig. 1 and for the calculation of the sensitivity. If all children in multiple pregnancies were $\mathrm{RhD}$ negative in multiple pregnancies, this was counted as "one RhD negative newborn" in Fig. 1 and for the calculation of the specificity. 1475 women delivered at least one RhD positive $(n=1462)$ or weak D child $(n=13)$ of which 1474 serological $\mathrm{RhD}$ results were predicted correctly, the sensitivity was $99.93 \%$ (95\% CI 99.61-99.99\%). A frozen backup sample of the single false-negative case, a singleton pregnancy at $13+4 \mathrm{wg}$, tested $R H D$ positive in a repeat test. In 769 cases, one or more $\mathrm{RhD}$ negative children were born. In three of these cases, NIPT RhD predicted an RhD positive newborn. The specificity of the FetoGnost RhD test was 99.61\% (95\% CI 98.86-99.87\%) and the accuracy 99.82\% (95\% CI 99.54-99.93\%). Even when results from samples collected beyond 19 wg were excluded (no errors in this cohort, $n=488$ ), a high sensitivity of $99.91 \%$ (95\% CI $99.50-99.98 \%, n=1144)$, specificity of $99.51 \%$ (95\% CI $98.57-99.83 \%, n=612)$ and accuracy of $99.77 \%(95 \% \mathrm{CI}$ 99.42-99.91, $n=1756$ ) was observed.

During the observation period, NIPT RhD was performed in 199 pregnancies with twins and in 7 pregnancies with triples. These cases are included in the numbers of Fig. 1

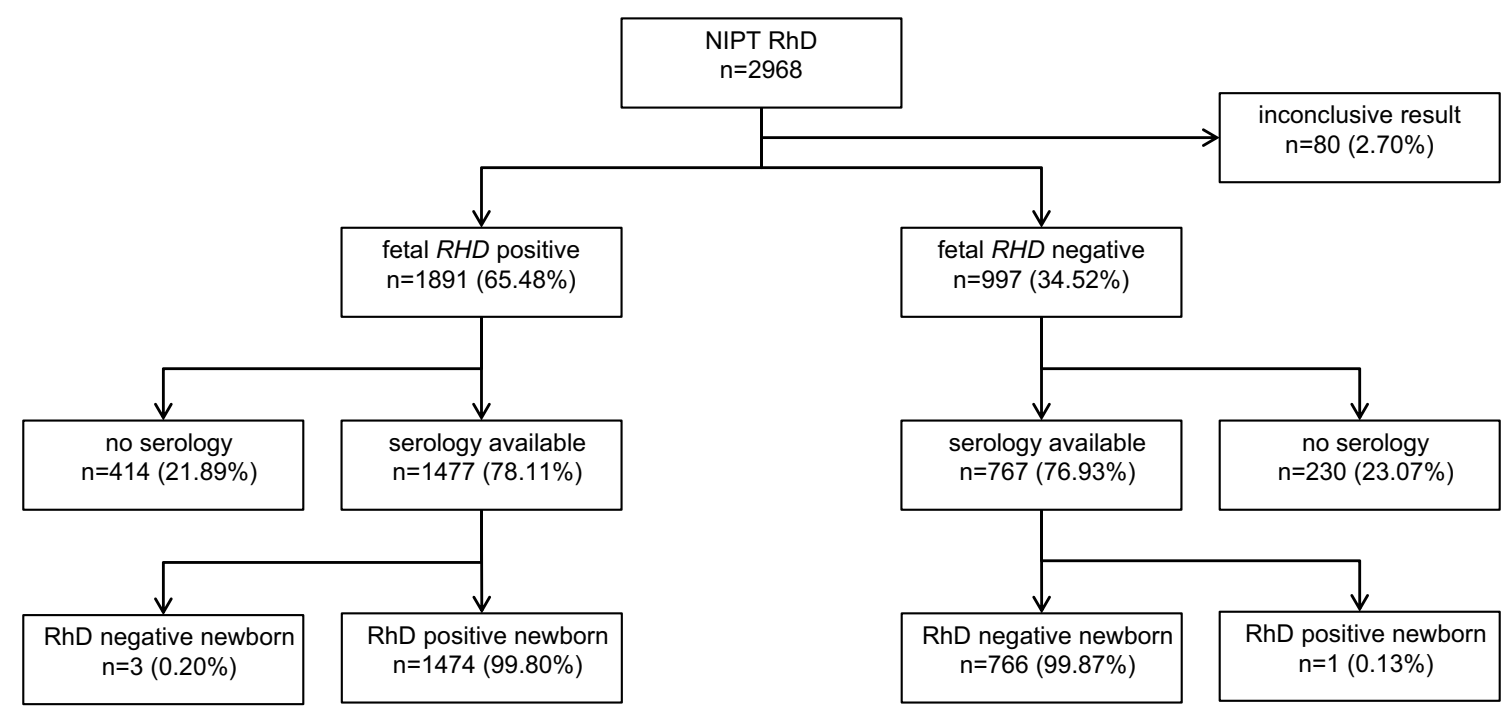

Fig. 1 Flow of participants. NIPT RhD was performed with the FetoGnost RhD assay taking fetal markers SRY and hypermethylate RASSF1A into account. The results of the newborn's RhD status serologically determined from cord blood were taken as a reference 
and in the calculation of sensitivity and specificity. In 205 (99.5\%) of these 206 cases, a conclusive NIPT RhD result was obtained and in 203 cases the phenotype of the newborns was reported. Complete concordance between NIPT $\mathrm{RhD}$ result and cord blood results was observed in 196 pregnancies with twins and in 7 pregnancies with triplets, respectively. In 141 cases, at least one child was RhD positive, in one case, both twins were weak $\mathrm{D}$, and in 61 cases, all children were $\mathrm{RhD}$ negative.

\section{Discussion}

Recently, data from about 60.000 study participants were pooled in a large meta-analysis for the purpose to determine the diagnostic accuracy of NIPT RhD [20]. However, in this systematic review, only studies were included, which evaluated lab developed (in-house) tests. In contrast, the literature dealing with the validation of commercial NIPT RhD test kits is less comprehensive. The SensiGene ${ }^{\circledR}$ RHD assay (Sequenom, San Diego, CA, USA) detects exons 4, 5 and 7 of the RHD gene, RHD psi and three sequences on the Y chromosome (SRY, TTTY, DBY) for the control of fetal DNA in male fetuses. Initially, Bombard et al. described a sensitivity of $97.2 \%$, and a specificity of $96.8 \%(n=207)$ of the SensiGene ${ }^{\circledR}$ RHD assay taking the newborn's serological $\mathrm{RhD}$ result as a reference. However, in comparison with genotype reference, they observed an increased sensitivity $(100.0 \%)$ and specificity $98.3 \%(n=199)$ [25]. In a subsequent observational study, Moise et al. found one false-negative result in $324 \mathrm{RhD}$ positive fetuses due to mislabeling of a collection tube and 2 false-positive results in $136 \mathrm{RhD}$ negative fetuses when testing between 11 and $29 \mathrm{wg}$ [26].

Protected by an exclusive patent license, the Free DNA Fetal Kit ${ }^{\circ}$ RhD (Institut de Biotechnologies Jacques Boy, Reims, France) was until recently the only real-time NIPT $\mathrm{RhD}$ PCR assay commercially available in Europe. In a validation study performed to receive CE-approval of this test kit, Roullica-Le Sciellour et al. described a sensitivity of $100 \%$ and a specificity of $>99 \%$ (two false-positive) in 300 plasma specimen tested between 10 and 34 weeks of gestation [27]. In addition, Londero et al. found more recently a complete concordance between the Free DNA Fetal Kit ${ }^{\circledR}$ $\mathrm{RhD}$ result obtained from week $11+6$ of gestation until term and $\mathrm{RhD}$ phenotype at birth in all 133 cases investigated [28]. In comparison with these studies, the FetoGnost RhD assay was evaluated with more pregnant women during this continuous performance evaluation in Vienna. Diagnostic accuracy of this test kit was as high as the diagnostic accuracy determined in larger nation-wide screening studies with in-house NIPT for RhD [14, 29, 30]. One falsepositive result could be explained by a maternal non-coding $R H D$ variant, in a second case, two fetuses vanished during a pregnancy with triplets and only an $\mathrm{RhD}$ negative fetus survived and for the third false-positive result no conclusive reason was found. In this case and in one false-negative case with a positive repeat test, either the first test was wrong due to a technical failure or a wrong sample was tested as a consequence of human error.

Beyond RAADP, anti-D administration is also required earlier in $\mathrm{RhD}$ negative pregnancy whenever there are clinical signs or risks for fetomaternal hemorrhage [8]. Therefore, we were especially interested in the sensitivity and specificity of the FetoGnost assay in the first trimester. Wikman and co-workers described in 4118 pregnancies an increase of sensitivity of a single-exon fetal $R H D$ assay during the course of pregnancy. After exclusion of samples analyzed before $10 \mathrm{wg}$, the sensitivity was $99.3 \%$ and it increased up to $100 \%$ when results from $22 \mathrm{wg}$ or later were included into the calculation [30]. In a large study performed in seven maternity units in England, Chitty et al. described a sensitivity of $99.83 \%, 99.67 \%, 99.82 \%$ and $100 \%$ at $11-13,14-17$, 18-23 and $>23$ completed wg, respectively. Although falsenegative results were rare, they were mainly observed earlier in gestation [31]. In contrast, another study performed in 10-14 wg did not observe false-negative results in 416 serum samples (2.2\% inconclusive). However, the specificity of an assay based on replicate testing of RHD exon 10 with $95.2 \%$ was lower than in the previous studies [32]. In our performance evaluation study, the sensitivity of $99.93 \%$ and specificity of $99.61 \%$ were as high as sensitivities and specificities determined in the second trimester with largescale screening studies [14, 21, 29, 33]. Notably, our single false-negative result was observed at the beginning of the second trimester.

In multiple pregnancies, no false-negative NIPT $\mathrm{RhD}$ results were described in 3 studies with overall 92 cases [30, 34, 35]. With this publication, we add another 203 cases, where NIPT for RhD correctly predicted the risk of anti-D alloimmunization if that at least one newborn was $\mathrm{RhD}$ positive or weak D, whereas no false-positive genotyping results were reported in 61 cases. Therefore, targeted RAADP is reasonable also in multiple pregnancies.

In a recent review Yang et al. concluded, that false-negative results are rather rare after $13 \mathrm{wg}$. [18]. Due to the falsenegative case at $13+4 \mathrm{wg}$ in our diagnostic accuracy study we support the conclusion of the German Association for Transfusion Medicine and Immunohematology (DGTI) that a single NIPT for RhD test beyond $19 \mathrm{wg}$ is a safe procedure to apply targeted RAADP in the second trimester [36]. If testing for fetal markers was not been performed in Vienna, one more false-negative case would have occurred at $11+3$ wg. Therefore, we suggest, that before $20 \mathrm{wg}$ a control for fetal DNA should confirm the presence of cff DNA if NIPT for $\mathrm{RhD}$ predicts an $\mathrm{RhD}$ negative fetus and if no second sample for confirmation is available due to public health 
economic considerations. The suitability of testing hypermethylated RASSF1A with real-time PCR as fetal marker has also been demonstrated by other groups [37-39].

In conclusion, NIPT RhD performed with the FetoGnost $\mathrm{RhD}$ assay delivers reliable results in the first and second trimester both in singleton and multiple pregnancies, respectively. However, even if an analytical process is $100 \%$ reliable through a maximum of automation, human errors during blood collection or labelling of blood tubes never can be completely excluded. Based on the analysis of underlying risks for the individual patient, a single NIPT for RhD can be considered sufficient for targeted anti-D prophylaxis. In contrast, if the management of an alloimmunized woman with antibodies against paternally inherited blood group antigens has to be stratified, a negative NIPT for RhD has to be confirmed from a second, independent blood drawing, because errors due to mislabeling of blood tubes can never be excluded.

Acknowledgements The support from all technicians involved in testing and data collection is greatfully acknowledged.

Author contributions All authors contributed to the study conception and design. Material preparation, data collection and analysis were performed by all authors. The first draft of the manuscript was written by Tobias Legler and Sandra Lührig. All authors commented on previous versions of the manuscript. All authors read and approved the final manuscript.

Funding Open Access funding enabled and organized by Projekt DEAL.

Data availability All authors made sure that all data and materials support the published claims of this manuscript and comply with field standards.

\section{Declarations}

Conflict of interest Tobias Legler receives consultation fees from LADR GmbH and participates in the revenue of his employer. Irina Korschineck is the owner and manager of the company Ingenetix $\mathrm{GmbH}$. Sandra Lührig and Dieter Schwartz do not have any conflicts of interest/competing interests to declare.

Ethical approval This study has been approved by the Ethics Committee of Vienna University (approval no. 1927/2020), the approval has been published: https://ekmeduniwien.at/vote/20798/download/.

Informed consent Before blood sample collection for NIPT-RhD, informed consent was obtained from all patients.

Consent for publication Not applicable, only anonymized data are reported.

Open Access This article is licensed under a Creative Commons Attribution 4.0 International License, which permits use, sharing, adaptation, distribution and reproduction in any medium or format, as long as you give appropriate credit to the original author(s) and the source, provide a link to the Creative Commons licence, and indicate if changes were made. The images or other third party material in this article are included in the article's Creative Commons licence, unless indicated otherwise in a credit line to the material. If material is not included in the article's Creative Commons licence and your intended use is not permitted by statutory regulation or exceeds the permitted use, you will need to obtain permission directly from the copyright holder. To view a copy of this licence, visit http://creativecommons.org/licenses/by/4.0/.

\section{References}

1. Hyland CA, O'Brien H, Flower RL, Gardener GJ (2020) Noninvasive prenatal testing for management of haemolytic disease of the fetus and newborn induced by maternal alloimmunisation. Transfus Apher Sci 59:102947

2. Finning K, Martin P, Summers J, Daniels G (2007) Fetal genotyping for the $\mathrm{K}$ (Kell) and Rh C, c, and E blood groups on cellfree fetal DNA in maternal plasma. Transfusion 47:2126-2133

3. Gutensohn K, Müller SP, Thomann K, Stein W, Suren A, Körtge-Jung S, Schlüter G, Legler TJ (2010) Diagnostic accuracy of noninvasive polymerase chain reaction testing for the determination of fetal rhesus $\mathrm{C}, \mathrm{c}$ and $\mathrm{E}$ status in early pregnancy. BJOG 117:722-729

4. Scheffer P, van der Schoot C, Page-Christiaens G, de Haas M (2011) Noninvasive fetal blood group genotyping of rhesus D, $\mathrm{c}, \mathrm{E}$ and of $\mathrm{K}$ in alloimmunised pregnant women: evaluation of a 7-year clinical experience. BJOG 118:1340-1348

5. O'Brien H, Hyland C, Schoeman E, Flower R, Daly J, Gardener G (2020) Non-invasive prenatal testing (NIPT) for fetal Kell, Duffy and Rh blood group antigen prediction in alloimmunised pregnant women: power of droplet digital PCR. Br J Haematol 189:e90-e94

6. Orzińska A, Guz K, Mikula M, Kluska A, Balabas A, Ostrowski J, Uhrynowska M, Kopeć I, Dębska M, Luterek K, Brojer E (2019) Prediction of fetal blood group and platelet antigens from maternal plasma using next-generation sequencing. Transfusion 59:1102-1107

7. Wienzek-Lischka S, Bachmann S, Froehner V, Bein G (2020) Potential of next generation sequencing in noninvasive fetal molecular blood group genotyping. Transfus Med Hemother 47:14-22

8. Qureshi H, Massey E, Kirwan D, Davies T, Robson S, Wjthe J, Jones J, Allard S (2014) BCSH guideline for the use of anti-D immunoglobulin for the prevention of haemolytic disease of the fetus and newborn. Transfus Med 24:8-20

9. Gudlaugsson B, Hjartardottir H, Svansdottir G, Gudmundsdottir G, Kjartansson S, Jonsson T, Gudmundsson S, Halldorsdottir AM (2020) Rhesus D alloimmunization in pregnancy from 1996 to 2015 in Iceland: a nation-wide population study prior to routine antenatal anti-D prophylaxis. Transfusion 60:175-183

10. Legler TJ (2020) RhIg for the prevention Rh immunization and IVIg for the treatment of affected neonates. Transfus Apher Sci 59:102950

11. Lo YM, Hjelm NM, Fidler C, Sargent IL, Murphy MF, Chamberlain PF, Poon PM, Redman CW, Wainscoat JS (1998) Prenatal diagnosis of fetal RhD status by molecular analysis of maternal plasma. N Engl J Med 339:1734-1738

12. Faas BH, Beuling EA, Christiaens GC, von dem Borne AE, van der Schoot CE (1998) Detection of fetal RhD-specific sequences in maternal plasma. Lancet 352:1196

13. Clausen FB, Christiansen M, Steffensen R et al (2012) Report of the first nationally implemented clinical routine screening for fetal $R H D$ in D-pregnant women to ascertain the requirement for antenatal RhD prophylaxis. Transfusion 52:752-758 
14. Clausen FB, Steffensen R, Christiansen M, Rudby M, Jakobsen MA, Jakobsen TR, Krog GR, Madsen RD, Nielsen KR, Rieneck K, Sprogøe U, Homburg KM, Baech J, Dziegiel MH, Grunnet N (2014) Routine noninvasive prenatal screening for fetal $R H D$ in plasma of RhD-negative pregnant women-2 years of screening experience from Denmark. Prenat Diagn 34:1000-1005

15. van der Schoot CE, de Haas M, Clausen FB (2017) Genotyping to prevent Rh disease: has the time come? Curr Opin Hematol 24:544-550

16. Saramago P, Yang H, Llewellyn A, Walker R, Harden M, Palmer $S$ et al (2018) High-throughput non-invasive prenatal testing for fetal rhesus D status in RhD-negative women not known to be sensitised to the RhD antigen: a systematic review and economic evaluation. Health Technol Assess 22:13

17. Zhu YJ, Zheng YR, Li L et al (2014) Diagnostic accuracy of noninvasive fetal RhD genotyping using cell-free fetal DNA: a meta analysis. J Matern Fetal Neonatal Med 27:1839-1844

18. Yang H, Llewellyn A, Walker R et al (2019) High-throughput, non-invasive prenatal testing for fetal rhesus $\mathrm{D}$ status in RhDnegative women: a systematic review and meta-analysis. BMC Med 17:37

19. van der Schoot CE, Winkelhorst D, Clausen FB (2018) Noninvasive fetal blood group typing. Noninvasive prenatal testing (NIPT). Applied genomics in prenatal screening and prenatal diagnosis. Page-Christiaens L, Klein H-G (Eds.).

20. Runkel B, Bein G, Sieben W, Sow D, Polus S, Fleer D (2020) Targeted antenatal anti-D prophylaxis for $\mathrm{RhD}$-negative pregnant women: a systematic review. BMC Pregnancy Childbirth 20:83. https://doi.org/10.1186/s12884-020-2742-4

21. Clausen FB (2020) Cell-free fetal DNA and fetal blood group genotyping: non-invasive prenatal testing. ISBT Sci Ser 15:46-51

22. Grootkerk-Tax MG, Soussan AA, de Haas M, Maaskant-van Wijk PA, van der Schoot CE (2006) Evaluation of prenatal RhD typing strategies on cell-free fetal DNA from maternal plasma. Transfusion 46:2142-2148

23. Legler TJ, Liu Z, Mavrou A, Finning K, Hromadnikova I, Galbiati S, Meaney C, Hultén MA, Crea F, Olsson ML, Maddocks DG, Huang D, Armstrong Fisher S, Sprenger-Haussels M, Ait Soussan A, van der Schoot CE (2007) Workshop report on the extraction of fetal DNA from maternal plasma. Prenat Diagn 27:824-829

24. Chan KC, Ding C, Gerovassili A, Yeung SW, Chiu RW, Leung TN, Lau TK, Chim SS, Chung GT, Nicolaides KH, Lo YM (2006) Hypermethylated Rassf1a in maternal plasma: a universal fetal DNA marker that improves the reliability of noninvasive prenatal diagnosis. Clin Chem 52:2211-2218

25. Bombard AT, Akolekar R, Farkas DH et al (2011) Fetal RHD genotype detection from circulating cell-free fetal DNA in maternal plasma in non-sensitized RhD negative women. Prenat Diagn 31:802-808

26. Moise KJ Jr, Gandhi M, Boring NH et al (2016) Circulating cellfree DNA to determine the fetal $R H D$ status in all three trimesters of pregnancy. Obstet Gynecol 128:1340-1346. https://doi.org/10. 1097/AOG.0000000000001741
27. Rouillac-Le Sciellour C, Serazin V, Brossard Y, Oudin O, Le Van KC, Colin Y, Guidicelli Y, Menu M, Cartron JP (2007) Noninvasive fetal RhD genotyping from maternal plasma. Use of a new developed free DNA fetal kit RhD. Transfus Clin Biol 14:572-577

28. Londero D, Stampalija T, Bolzicco D et al (2019) Fetal RHD detection from circulating cell-free fetal DNA in maternal plasma: validation of a diagnostic kit using automatic extraction and frozen DNA. Transfus Med 29:408-414. https://doi.org/10.1111/tme. 12605

29. De Haas M, Thurik FF, van der Ploeg CPB et al (2016) Sensitivity of fetal RHD screening for safe guidance of targeted anti-D immunoglobulin prophylaxis: prospective cohort study of a nationwide programme in the Netherlands. BMJ 355:i5789

30. Wikman AT, Tiblad E, Karlsson A et al (2012) Noninvasive single-exon fetal $R H D$ determination in a routine screening program in early pregnancy. Obstet Gynecol 120:227-234

31. Chitty LS, Finning K, Wade A et al (2014) Diagnostic accuracy of routine antenatal determination of fetal RHD status across gestation: population based cohort study. BMJ 349:g5243

32. Vivanti A, Benachi A, Huchet FX et al (2016) Diagnostic accuracy of fetal rhesus D genotyping using cell-free fetal DNA during the first trimester of pregnancy. Am J Obstet Gynecol 215:606. e1-606.e5

33. Haimila K, Sulin K, Kuosmanen M et al (2017) Targeted antenatal anti-D prophylaxis program for $\mathrm{RhD}$-negative pregnant womenoutcome of the first two years of a national program in Finland. Acta Obstet Gynecol Scand 96:1228-1233

34. Müller SP, Bartels I, Stein W, Emons G, Gutensohn K, Köhler M, Legler TJ (2008) The determination of the fetal RhD status from maternal plasma for decision making on Rh prophylaxis is feasible. Transfusion 48:2292-2301

35. Minon JM, Gerard C, Senterre JM et al (2008) Routine fetal RHD genotyping with maternal plasma: a four-year experience in Belgium. Transfusion 48:373-381

36. Legler T, Bein G, Bugert P, Schwartz D (2020) Validierung von Testverfahren zur Bestimmung des fetalen RHD-Status aus dem Blut D-negativer Frauen in der Schwangerschaft. Transfusionsmedizin 20:50-54

37. Hyland CA, Gardener GJ, Davies H et al (2009) Evaluation of non-invasive prenatal RHD genotyping of the fetus. Med J Aust 191:21-25

38. Zejskova L, Jancuskova T, Kotlabova K, Doucha J, Hromadnikova I (2010) Feasibility of fetal-derived hypermethylated RASSF1A sequence quantification in maternal plasma-next step toward reliable non-invasive prenatal diagnostics. Exp Mol Pathol 89:241-247

39. White HE, Dent CL, Hall VJ, Crolla JA, Chitty LS (2012) Evaluation of a novel assay for detection of the fetal marker RASSF1A: facilitating improved diagnostic reliability of noninvasive prenatal diagnosis. PLoS ONE 7:e45073

Publisher's Note Springer Nature remains neutral with regard to jurisdictional claims in published maps and institutional affiliations. 